ACCEPTED MANUSCRIPT

\title{
Influence of temperature on the magnetic oscillations in graphene with spin splitting: a new approach
}

To cite this article before publication: Federico Nahuel Escudero et al $2018 \mathrm{~J}$. Phys.: Condens. Matter in press https://doi.org/10.1088/1361$\underline{648 X / a a c 7 e a}$

\author{
Manuscript version: Accepted Manuscript \\ Accepted Manuscript is "the version of the article accepted for publication including all changes made as a result of the peer review process, \\ and which may also include the addition to the article by IOP Publishing of a header, an article ID, a cover sheet and/or an 'Accepted \\ Manuscript' watermark, but excluding any other editing, typesetting or other changes made by IOP Publishing and/or its licensors" \\ This Accepted Manuscript is @ 2018 IOP Publishing Ltd.
}

During the embargo period (the 12 month period from the publication of the Version of Record of this article), the Accepted Manuscript is fully protected by copyright and cannot be reused or reposted elsewhere.

As the Version of Record of this article is going to be / has been published on a subscription basis, this Accepted Manuscript is available for reuse under a CC BY-NC-ND 3.0 licence after the 12 month embargo period.

After the embargo period, everyone is permitted to use copy and redistribute this article for non-commercial purposes only, provided that they adhere to all the terms of the licence https://creativecommons.org/licences/by-nc-nd/3.0

Although reasonable endeavours have been taken to obtain all necessary permissions from third parties to include their copyrighted content within this article, their full citation and copyright line may not be present in this Accepted Manuscript version. Before using any content from this article, please refer to the Version of Record on IOPscience once published for full citation and copyright details, as permissions will likely be required. All third party content is fully copyright protected, unless specifically stated otherwise in the figure caption in the Version of Record.

View the article online for updates and enhancements. 


\title{
Influence of temperature on the magnetic oscillations in graphene with spin splitting: a new approach
}

\author{
F Escudero, J S Ardenghi, P Jasen
}

IFISUR, Departamento de Física (UNS-CONICET), Av. Alem 1253, B8000CPB

Bahía Blanca, Argentina

Instituto de Física del Sur (IFISUR, UNS-CONICET)

Av. Alem 1253, B8000CPB Bahía Blanca, Argentina

E-mail: federico.escudero@uns.edu.ar

\begin{abstract}
We analyze the magnetic oscillations (MO) due to the de Haas-van Alphen effect, in pristine graphene under a perpendicular magnetic field, taking into account the Zeeman effect. We consider a constant Fermi energy, such that the valence band is always full and only the conduction band is available. At zero temperature the MO consist of two sawtooth peaks, one for each spin. Both peaks have the same frequency, but different amplitude and phase. We show that, in order to observe the spin splitting in the MO, Fermi energy of about $0.1 \mathrm{eV}$ is required. At low temperatures we obtain that the MO can be expressed as the MO at zero temperature, plus small FermiDirac like functions, each centered/around the MO peaks. Using this expression, we show that the spin splitting is observable in the MO only when the thermal energy is smaller than the Zeeman energy. We also analyze the shift of the MO extrema as the temperature increases. We show that it depends on the magnetic field, which implies a broken periodicity at nonzero temperature. Finally, we obtain an analytical expression for the MO envelope. The results obtained could be used to infer temperature changes from the MO extrema shift and vice versa.
\end{abstract}




\section{Magnetic oscillations in graphene with spin splitting: a new approach}

\section{Introduction}

Since its experimental isolation in 2004, graphene has become one of the most interesting and promising materials in condensed matter science [1, 2, 3]. Its unique features, like high heat and electrical conductivity, has make graphene an exciting matérial for technological applications [4, 5]. These properties are related to its 2D hexagonal structure, composed of two interpenetrating sublattices A and B [6]. Without impurities or defects, the conduction and valence bands touch at the Fermi energy, with the valence band full and the conduction band empty in the ground state [7]. Furthermore, in pristine graphene the density of states at the Fermi energy is zero, making graphene a semiconductor with zero band gap, or a semi-metal [8]. In the long wavelength approximation the dispersion relation is relativistic and the electrons behave as massless fermions, moving with a Fermi velocity $\sim 10^{6} \mathrm{~m} / \mathrm{s}[9]$.

The magnetic properties of graphene and $2 \mathrm{D}$ systems have been investigated in recent works [10, 11, 12, 13, 14]. Unlike conventional materials, the magnetization in graphene has unique features. This can be related to the Landau levels (LL) that appear when a magnetic field is applied [15]. In a classical system, these LL are equidistant due to the parabolic dispersion relation. But in graphene, at low energies the dispersion relation is relativistic [7], causing the LL to be not equidistant, which in turn affects the oscillating behavior in the thermodynamics potentials [10]. For instance, the magnetic oscillations (MO), the so called de Haas van Alphen effect [16], are sawtooth at zero temperature [17, 18], with the peaks being caused by the change in the last occupied energy level [19].

The MO are altered by impurities and temperature. In each case the MO are broadened as a result of the modified density of states (DOS) and the Fermi-Dirac distribution. In classical metals this is described by the Lifshitz-Kosevich formula [20], which incorporates the effects of impurities and temperatures, as well as the spin. The essential feature of this formula is that the MO are written as an infinite series, where the damping effects are taken into account by the introduction of reduction factors. This formula has been extended to the case of graphene [17], where the difference only lies in the form the reduction factors take. In principle, this formula would be sufficient to describe the $\mathrm{MO}$ in the most general case, but due to its complexity it is not very useful. Thus, in general, limit cases are considered. The most familiar one is zero temperature an energy independent impurities, where the only reduction factor is the Dingle factor, in which case the infinite series can be easily evaluated. Another interesting situation is the pristine case (i.e. without impurities) at nonzero temperature. In this case the general series cannot yet be evaluated, due to the complicated temperature reducing factor, so in general some approximations are considered. The most prominent one is the limit $B \rightarrow 0$ (low magnetic field), where one can simplify the temperature reduction factor and easily evaluate the infinite series. Nevertheless, one could argue that this limit may not be very useful, since the lower the magnetic field the more difficult is to observe the MO. 


\section{Magnetic oscillations in graphene with spin splitting: a new approach}

When the Zeeman effect is considered, a splitting of the Landau levels appears, which is relevant for several thermodynamical properties [19]. In turn, when a gate voltage is applied, the electron density can be changed, which could be useful for spin filtering [21], spin-polarized currents in 2D systems [22] and spin conductivity [23]. Moreover, when other effects are considered, such as spin-orbit coupling due to an external electric field, the MO changes drastically [24] and the interplay between the degeneracy of each level, the disorder and spin-orbit coupling is not trivial [25].

Motivated by this, we analyzed the MO in pristine graphene, in the presence of a perpendicular magnetic field, taking into account the Zeeman effect. We have organized this work as follow: in section 2 we study the MO in graphene at zero temperature, taking into the account the Zeeman effect. We then analyze the conditions to observe the spin splitting in the MO. In section 3 we study the MO at nonzero temperature, where we make an approximation to obtain an useful analytical formula for the magnetization. From this we analyze how the temperature affects the observation of the spin splitting in the MO. We end with a study of the maxima and minima shift in the MO as a function of the temperature, from which we obtain an expression for the oscillation envelope. Finally, our conclusions follow in section 4.

\section{MO at zero temperature}

In order to study the MO, we will consider that the Fermi energy $\mu$ is held constant. Moreover, we shall always take $\mu>0$, so that the valence band is always full. Given that $\mu$ is fixed, while the number of electrons $N$ may change, it is convenient to work with the grand potential $\Omega$. We will consider the long wavelength approximation, with energies close to the Fermi level. This condition is satisfied as long as [7] $\mu \ll|t| \sim 3$ $\mathrm{eV}$ ( $t$ is the nearest neighbor hopping amplitude). In this case the electrons in graphene behave as relativistic massless fermions, whose dynamics is given by the corresponding Dirac Hamiltonian. In the presence of a perpendicular magnetic field $B$ one obtains the discrete Landau level, as well as a spin splitting due to the Zeeman effect [26]. Then it can be shown [19] that in graphene the energy levels are given by $\varepsilon_{\lambda, n, s}=\lambda \alpha \sqrt{n B}-s \mu_{\mathrm{B}} B$, where $\alpha=v_{F} \sqrt{2 e \hbar}$ and $v_{F} \sim 10^{6} \mathrm{~m} / \mathrm{s}$ is the Fermi velocity, while $\lambda= \pm 1$ for the conduction and valence bands, $n=0,1,2, \ldots$ for the Landau level (LL) and $s= \pm 1$ for the spin (throughout this paper we will use +1 to indicate spin up and -1 to indicate spin down). Each energy level has a degeneracy given by $D=2 \mathcal{A} B / \phi$, where $\mathcal{A}$ is the sheet area of graphene, $\phi=h / e$ is the magnetic unit flux and the factor of 2 takes into account the valley degeneracy [10].

For a Fermi energy $\mu>0$ at zero temperature, the valence band is full while the conduction band is partially filled. We will write the conduction energy levels as $\varepsilon_{m}=\alpha \sqrt{n_{m} B}-s_{m} \mu_{\mathrm{B}} B$, where we have introduced the decreasing energy sorting index $m=0,1,2, \ldots$, so $n_{m}$ gives the LL and $s_{m}$ the spin for the $m$ position. In general, the mixing of the LL depends on the magnetic field [27], but for usual values there is no spin mixing so $n_{m}=\sqrt{m / 2-\left[1-(-1)^{m}\right] / 4}$ and $s_{m}=(-1)^{m}$. At a given $\mu>0$, all 
Magnetic oscillations in graphene with spin splitting: a new approach

conduction energy levels $m=0,1,2, \ldots, f$ are filled, where $f$ is such that $\varepsilon_{f}<\mu \leq \varepsilon_{f+1}$. Then the grand potential at zero temperature is

$$
\Omega=\Omega_{V}+\sum_{m=0}^{f} D\left(\varepsilon_{m}-\mu\right),
$$

where $\Omega_{V}$ is the grand potential due to the filled valence band. From equation (1) we shall expect the oscillatory contribution coming only from the last term, associated with the change in the last energy level as $B$ is modified. On the other hand, the first term $\Omega_{V}$ makes a non-oscillatory contribution since the valence band is always full if $\mu>0$. Therefore we omit for the moment $\Omega_{V}$ and consider only the conduction grand potential $\Omega_{C}=\sum_{m=0}^{f} D\left(\varepsilon_{m}-\mu\right)$. Separating $\varepsilon_{m}=\varepsilon_{m}^{0}-s_{m} \mu_{\mathrm{B}} B$, with $\varepsilon_{m}^{0}=\alpha \sqrt{n_{m} B}$, we get

$$
\Omega_{C}=\Omega_{0}-D \mu_{\mathrm{B}} B \sum_{m=0}^{f} s_{m},
$$

where $\Omega_{0}=\sum_{m=0}^{f} D\left(\varepsilon_{m}^{0}-\mu\right)$. The last term in equation 2 2 is related to the Pauli paramagnetism $M_{P}$, associated with the spin population. Indeed, we have $D \sum_{m=0}^{f} s_{m}=N_{+}-N_{-}$, where $N_{+}\left(N_{-}\right)$is the total number of spin up(down) states. Thus $\mu_{\mathrm{B}} D \sum_{m=0}^{f} s_{m}=\mu_{\mathrm{B}} D\left[1+(-1)^{f}\right] / 2=\mu_{\mathrm{B}}\left(N_{+}-N_{-}\right)=M_{p}$, so equation 2 becomes

$$
\Omega_{C}=\Omega_{0}-B M_{P}
$$

The conduction magnetization is given by $M_{C}=-\mathcal{A}^{-1}\left(\partial \Omega_{C} / \partial B\right)_{\mu}$, where $\mathcal{A}$ is the graphene area. From equation (3) we gét

$$
M_{C}=M_{0}+\frac{1}{\mathcal{A}}\left(M_{P}+B \frac{\partial M_{P}}{\partial B}\right),
$$

where $M_{0}=-\mathcal{A}^{-1}\left(\partial \Omega_{0} / \partial B\right)_{\mu}$. Given that $\partial D / \partial B=D / B$, while $\partial \varepsilon_{m}^{0} / \partial B=\varepsilon_{m}^{0} / 2 B$, we can write

$$
M_{C}=-\frac{1}{2 B}\left(\frac{3 \Omega_{C}}{\mathcal{A}}+n \mu\right)+\frac{1}{2} m_{P},
$$

where $n=N / \mathcal{A}=\sum_{m=0}^{f} D / \mathcal{A}=D(f-1) / \mathcal{A}$ is the density of conduction electrons and $m_{p}=M_{P} / \mathcal{A}$. Equation (5) shows that the MO have a sawtooth oscillation (SO) produced whenever $n$ or $m_{P}$ changes discontinuously, $\Omega_{C}$ being continuous always. The sawtooth peaks amplitude $\Delta M$ are given by

$$
\Delta M=-\frac{\mu}{2 B} \Delta n+\frac{1}{2} \Delta m_{P}
$$

Each contribution $\Delta n$ and $\Delta m_{P}$ is determined by the discontinuous change in the parameters $n_{f}$ and $s_{f}$, which define the last energy level occupied. Considering the possible changes of LL and spin, we can write the MO as a sum of two sawtooth. In general the period of oscillation is given by $\Delta(1 / B)=1 / B_{2}-1 / B_{1}$, where $B_{i}$ is such 
Magnetic oscillations in graphene with spin splitting: a new approach

that $\varepsilon_{f_{i}}\left(B_{i}\right)=\mu$. Therefore $\mu=\alpha \sqrt{B_{i} n_{i}}-s_{i} \mu_{\mathrm{B}} B_{i}$, where for simplicity we have noted $n_{f_{i}}=n_{i}$ and $s_{f_{i}}=s_{i}$. Using the approximation $\mu^{2}+2 s_{i} \mu \mu_{\mathrm{B}} B_{i}+\mu_{\mathrm{B}}^{2} B_{i}^{2} \simeq \mu^{2}+2 s_{i} \mu \mu_{\mathrm{B}} B_{i}$, which holds for typical values of magnetic field, we obtain the $B_{i}$ at which the peaks occur:

$$
\frac{1}{B_{i}}=\frac{n_{i} \alpha^{2}}{\mu^{2}}-\frac{2 s_{i} \mu_{\mathrm{B}}}{\mu} .
$$

We shall consider that the two peaks correspond to a fixed spin, with the oscillation being given when the LL changes by one. Thus we take $\Delta n=n_{2}-n_{1}=1$, while $s=s_{1}=s_{2}$. Therefore, from equation (7) we obtain the period $\Delta(1 / B)$ and frequency $\omega=[\Delta(1 / B)]^{-1}$

$$
\omega_{s}=\frac{\mu^{2}}{\alpha^{2}}
$$

Then we can write equation (7) as $1 / B_{i, s}=n_{i} / \omega_{s}+\Delta_{s}$, where $\Delta_{s}$ is the phase

$$
\Delta_{s}=-\frac{2 s_{i} \mu_{\mathrm{B}}}{\mu}
$$

From equations (8) and (9) we see that $\omega_{1}=\omega_{-1}$ and $\Delta_{1}=-\Delta_{-1}$. This means that the two sawtooth peaks have the same frequency but different phase. The peaks amplitude are obtained from equation (6). Suppose the magnetic field is increased so the last sorted position $f$ changes to $f-1$. For $\Delta n$ and $\Delta m_{P}$ we easily get $\Delta n=D / \mathcal{A}=2 B / \phi$ and $\Delta m_{P}=D \mu_{\mathrm{B}} s_{f} / \mathcal{A}=2 B \mu_{\mathrm{B}} s_{f} / \phi$, so in general

$$
A_{s}=\frac{-\mu+s \mu_{\mathrm{B}} B}{\phi}
$$

We are now in position to express the two $\mathrm{SO}$ as an infinite series, whose amplitude, frequency and phase are given by equations (8), (9) and (10). We shall note $\omega \equiv \omega_{1}=$ $\omega_{-1}=\mu^{2} / \alpha^{2}$ and $\Delta \equiv-\Delta_{1}=\Delta_{-1}=2 \mu_{\mathrm{B}} / \mu$. Then we have

$$
M_{S O}=\sum_{s= \pm 1} A_{s} \sum_{p=1}^{\infty} \frac{1}{\pi p} \sin \left[2 \pi p \omega\left(\frac{1}{B}+s \Delta\right)\right] .
$$

Equation (11) gives the SO contribution to the MO. There is still another oscillatory contribution, which comes from the continuous oscillation in $\Omega_{C}$. This oscillation is not a SO, but an expression for it can be straightforward obtained noticing that $M_{\text {osc }}=-\mathcal{A}^{-1}\left(\partial \Omega_{C}^{o s c} / \partial B\right)_{\mu}$. From equation (11) we get that $\Omega_{C}^{o s c}$ should be of the form $\Omega_{C}^{o s c}=\sum_{s= \pm 1} C_{s} \sum_{p=1}^{\infty}(\pi p)^{-2} \cos \left[2 \pi p \omega\left(\frac{1}{B}+s \Delta\right)\right]$, where $C_{s}$ satisfies $M_{o s c}=$ $-\mathcal{A}^{-1}\left(\partial \Omega_{C}^{o s c} / \partial B\right)_{\mu}$, so $2 \omega C_{s} / \mathcal{A} B^{2}=-A_{s}$. Therefore the MO is given by

$$
\begin{aligned}
M_{o s c}=\sum_{s= \pm 1}\{ & {\left[\frac{B}{\omega \phi}\left(-\mu+\frac{3}{2} s \mu_{\mathrm{B}} B\right)\right] \sum_{p=1}^{\infty} \frac{1}{(\pi p)^{2}} \cos \left[2 \pi p \omega\left(\frac{1}{B}+s \Delta\right)\right] } \\
& \left.+A_{s} \sum_{p=1}^{\infty} \frac{1}{\pi p} \sin \left[2 \pi p \omega\left(\frac{1}{B}+s \Delta\right)\right]\right\}
\end{aligned}
$$




\section{Magnetic oscillations in graphene with spin splitting: a new approach $\quad 6$}
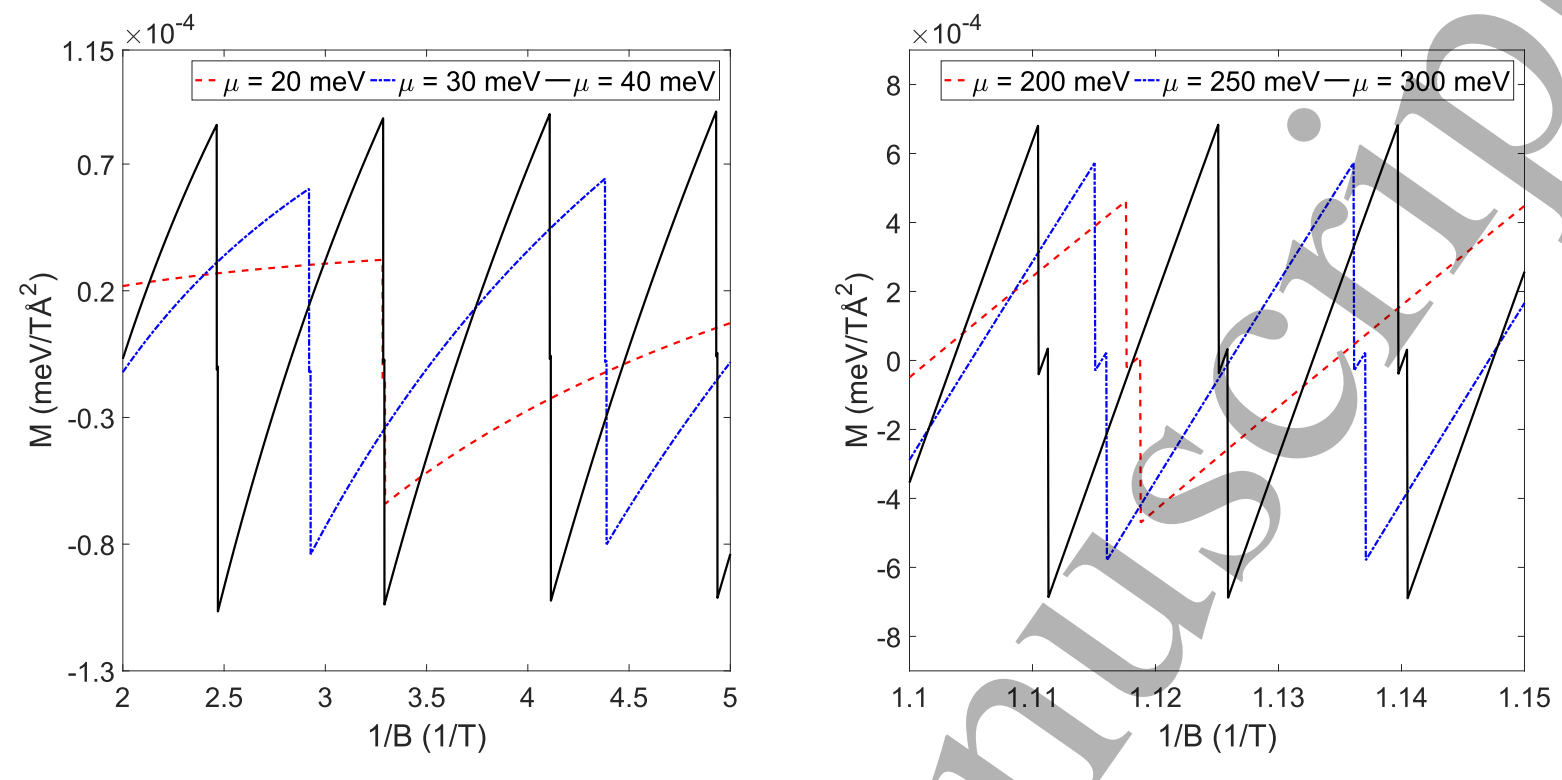

Figure 1. Magnetization given by equation 12, for (a) low $\mu$ and (b) high $\mu$, where $\mu$ is the Fermi energy.

Equation (12) is in agreement with the result found in [17], where the oscillating part of the magnetization is expressed as an infinite series. To obtain the total magnetization we also have to add the non-oscillatory contribution from both the valence and conduction band. Nevertheless, it can be shown [17] that in graphene, at $\mu>0$, this contribution cancels and the total magnetization is just given by equation (12). This result can be intuitively understood by noticing that the non-oscillatory contribution to $M$ comes from the variation of the degeneracy and energy as $B$ is changed (which gives a variation proportional to $\sqrt{B}$, for $D \varepsilon \sim B^{3 / 2}$ and thus $\delta D \varepsilon \sim B^{1 / 2}$ ). Ignoring the Zeeman splitting, the valence and conduction band energy levels are equal with opposite sign, so their total contribution is canceled. Consequently, in order to study the magnetization in graphene at $\mu>0$ it is sufficient to work with equation 12 . It is worth noting that in this formalism, the spin splitting due to the Zeeman effect is already taken into account in equation (12), so there is no need to introduce it as a reduction factor. The spin splitting is usually neglected in the MO calculations, but we will see that it can have a noticeable effect in $M$ at high Fermi energy, with sufficient low temperature and/or high magnetic field.

\subsection{Spin splitting in the MO at zero temperature}

We shall now analyze equation (12) in more detail. First, notice that each spin has a different amplitude $A_{s}$, although from equation (10) we have $\left|A_{1}-A_{-1}\right|=\mu_{\mathrm{B}} B / \phi$, which usually is very small. On the other hand, the MO are periodic in $1 / B$ (as in the classical case), each spin peak with the same frequency $\omega=\mu^{2} / \alpha^{2}$, but with a phase difference $2 \Delta=4 \mu_{\mathrm{B}} / \mu$. These features can be seen in figure 1 , where the MO given by equation (12) is plot for (a) low $\mu$ and (b) high $\mu$. We observe that, in order 


\section{Magnetic oscillations in graphene with spin splitting: a new approach}

of magnitude, for $\mu \sim 10 \mathrm{meV}$ the $\mathrm{MO}$ are practically seen as one unique oscillation, while for $\mu \sim 100 \mathrm{meV}$ the spin splitting becomes noticeable. Moreover, we observe that at low $\mu$ there is a small curvature in the MO, which disappears at high $\mu$. This behavior can be explained by taking the ratio of amplitudes of both series, given by $A_{s}^{\cos }=B\left(-\mu+3 s \mu_{\mathrm{B}} B / 2\right) / \omega \phi$ and $A_{s}^{s i n}=\left(-\mu+s \mu_{\mathrm{B}} B\right) / \phi$. Considering that usually $B \mu_{\mathrm{B}} / \mu \ll 1$, we get $A_{s}^{\text {cos }} / A_{s}^{\text {sin }} \simeq B / \omega$. Thus, if $\mu>0.1 \mathrm{eV}$ we have $\omega=\mu^{2} / \alpha^{2}>10 \mathrm{~T}$ and $A_{s}^{\text {cos }} / A_{s}^{\text {sin }}<0.1 B[\mathrm{~T}]$, so unless $B$ is very high we have $A_{s}^{\text {cos }} / A_{s}^{\text {sin }} \ll 1$. For smaller values of $\mu$ this would not be case, and for big $B$ one may even have $A_{s}^{\cos } / A_{s}^{\sin }>1$. This is seen in figure 1(a), where at low $\mu$ the cosine series in equation (12) produces the small curvature in the MO. Nevertheless, we will not take $\mu$ this low for then the Zeeman effect becomes unobservable. Therefore, given that we will be mainly interested with the spin splitting in the MO, which is seen at high $\mu$, we shall directly neglect the first term in equation (12). Then the MO reduce to equation (11) and the sine series can be evaluated to obtain

$$
M=\sum_{s= \pm 1} \frac{A_{s}}{\pi} \arctan \left\{\cot \left[\pi \omega\left(\frac{1}{B}+s \Delta\right)\right]\right\} .
$$

We can calculate the amplitude of the spin splitting effect in the MO by taking into account the low point of the first peak and the high point of the second peak, as schematically indicated in figure 2. We consider the two peaks corresponding to a LL $l$, so that the first peak is located at $1 / B_{1}=l / \omega-\Delta$, while the second peak at $1 / B_{2}=l / \omega+\Delta$. Then, from equation (13) we get that at the low point of the first peak we have $M_{1}=A_{1} / 2-A_{-1} \arctan [\cot (2 \pi \omega \Delta)] / \pi$, while at the high point of the second peak we have $M_{2}=A_{1} \arctan [\cot (2 \pi \omega \Delta)] / \pi-A_{-1} / 2$. Thus the amplitude of splitting $\Delta M_{S}$ is given by

$$
\Delta M_{s}=A_{+}\left(1-\frac{2}{\pi} \arctan [\cot (2 \pi \omega \Delta)]\right),
$$

where $A_{+} \equiv\left|A_{1}+A_{-1}\right| / 2=\mu / \phi$. A better measurement of the effect is to take the relation between $\Delta M_{s}$ and the amplitude of the peaks, which can be approximated as $A_{+}$(because $A_{1} \simeq A_{-1}$ ). On the other hand, we can also analyze the splitting peak width, which is given by the phase difference $2 \Delta=4 \mu_{\mathrm{B}} / \mu$. This width also affect the visualization of the spin splitting in $M$, for at low values of Fermi energy, $2 \Delta$ becomes insignificant. Then we can compare this width with the oscillation period, given by $1 / \omega=\alpha^{2} / \mu^{2}$. In figure 3 it is plotted $\Delta M_{s} / A_{+}$and $2 \Delta \omega$ as a function of the Fermi energy. We see that for $\mu \lesssim 100 \mathrm{meV}$ we have $\Delta M_{s} / A_{+} \sim 10^{-2}$, while $2 \Delta \omega$ is also very small, which makes the spin splitting effect practically unobservable. But when we get to higher Fermi energies, about $\mu \gtrsim 250 \mathrm{meV}$, we have $\Delta M_{s} / A_{+} \sim 0.1$ so the effect starts to become more evident. Consequently, in order to account for the Zeeman effect in $M$ we will take Fermi energies above $200 \mathrm{meV}$. Notice also that $\Delta M_{s} / A_{+}$does not depend on $B$, so the effect of the Zeeman splitting in the magnetization at zero temperature is independent of the magnetic field. Nevertheless, we shall see that the 
Magnetic oscillations in graphene with spin splitting: a new approach

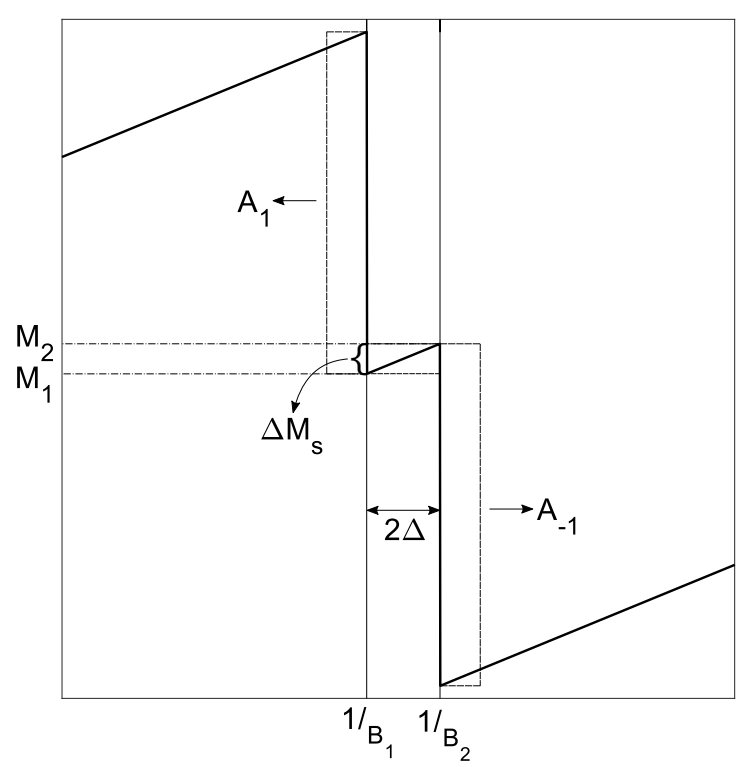

Figure 2. Scheme of two peaks due to the Zeeman effect, located at $1 / B_{1}=l / \omega-\Delta$ and $1 / B_{2}=l / \omega+\Delta$. We define the amplitude of splitting $\Delta M_{s}$ as shown, which measures the observation of the Zeeman effect in the MO. Notice also that the peaks have a phase difference $2 \Delta=4 \mu_{\mathrm{B}} / \mu$.

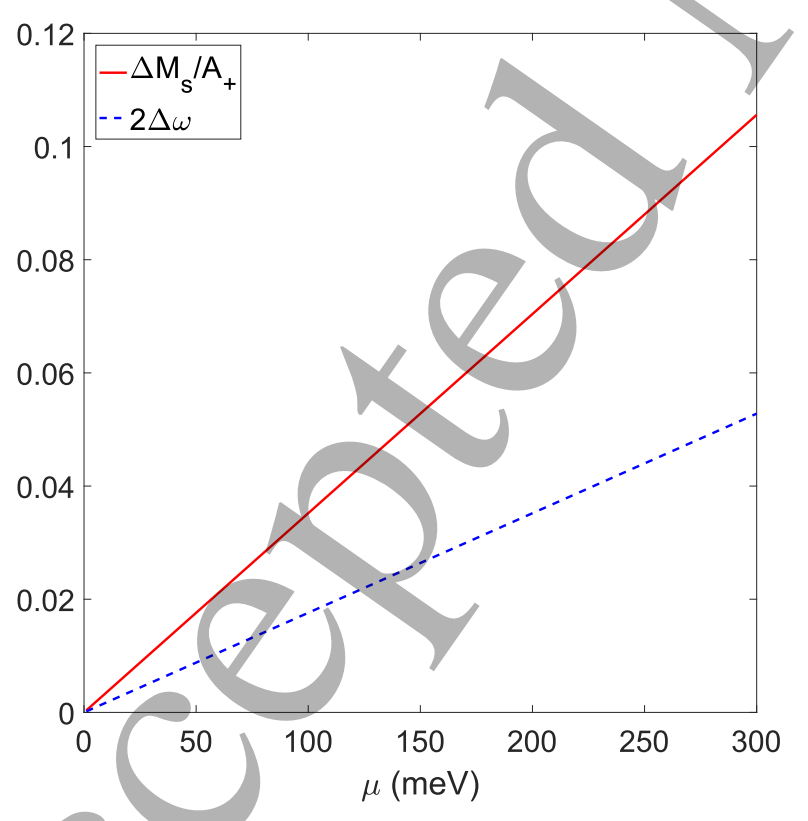

Figure 3. Plot of the parameters $\Delta M_{s} / A_{+}$and $2 \Delta \omega$ as a function of the Fermi energy, where $\Delta M_{s}$ is given by equation (14) and $A_{+} \equiv\left|A_{1}+A_{-1}\right| / 2=\mu / \phi$. The phase $\Delta$ and frequency $\omega$ are given by equations $(8)$ and $(9)$.

temperature makes the observation of the Zeeman splitting in the magnetization be strongly dependent of $B$. 
Magnetic oscillations in graphene with spin splitting: a new approach

\section{MO at nonzero temperature}

The effects of nonzero temperature can be taken into account in equation (11) by introducing the temperature reduction factor $R_{T}$, which for these systems has the form [17]

$$
R_{T}=\frac{4 \pi^{2} \mu p k T / \alpha^{2} B}{\sinh \left(4 \pi^{2} \mu p k T / \alpha^{2} B\right)}
$$

where $\alpha=v_{F} \sqrt{2 e \hbar}$. Thus the MO at nonzero temperature becomes

$$
M_{T}=\sum_{s= \pm 1} A_{s} \sum_{p=1}^{\infty} \frac{R_{T}}{\pi p} \sin \left[2 \pi p \omega\left(\frac{1}{B}+s \Delta\right)\right]
$$

In general, the series given by equation (16) cannot be evaluated, but it can be simplified under some approximations. The usual one is to take the limit $B \rightarrow 0$, in which case $1 / \sinh \left(4 \pi^{2} \mu p k T / \alpha^{2} B\right) \sim \exp \left(-4 \pi^{2} \mu p k T / \alpha^{2} B\right)$. Nevertheless, this limit implies low magnetic field, which in turn makes it difficult to observe the MO. Instead we will impose the low temperature limit, but such that the MO are still observable.

To obtain an approximation for $M_{T}$ at low temperatures, it is convenient to start from the expression of the grand potential $\Omega$ at $T \neq 0$ and $\mu>0$. It can be shown that in the absence of impurities, one can use the non-relativistic grand potential to obtain the magnetization [28]. Hence we start with

$$
\Omega_{T}=-k T \int_{-\infty}^{\infty} \rho_{0}(\varepsilon) \ln \left[1+e^{\beta(\mu-\varepsilon)}\right] d \varepsilon,
$$

where $\beta=1 / k T$ and $\rho_{0}(\varepsilon)=D \sum_{m} \delta\left(\varepsilon-\varepsilon_{m}\right)$ is the density of states (DOS) in the pristine case, where the summation is to be done considering the valence and conduction band. In this way we can separate the contribution from both bands, so that $\Omega_{T}=\Omega_{V, T}+\Omega_{C, T}$, where in general $\Omega_{T}=-k T \sum_{\varepsilon} D \ln \left[1+e^{\beta(\mu-\varepsilon)}\right]$. Now, for $\mu>0$ and low temperature we always have $\beta(\mu-\varepsilon) \gg 1$ for the valence band, so $\Omega_{V, T} \simeq \Omega_{V}(T=0)$ and $\partial \Omega_{V, T} / \partial B \simeq \partial \Omega_{V}(T=0) / \partial B$. Hence the valence band magnetization is not affected by the temperature under this conditions. Therefore, we shall omit it for the moment and continue with the conduction band grand potential

$$
\Omega_{C, T}=-k T \sum_{m=0}^{\infty} D \ln \left[1+e^{\beta\left(\mu-\varepsilon_{m}\right)}\right]=\sum_{m=0}^{\infty} \Omega_{T}^{m},
$$

where $\varepsilon_{m}=\alpha \sqrt{n_{m} B}-s_{m} \mu_{\mathrm{B}} B$ are the conduction energy levels, and we defined $\Omega_{T}^{m}=-k T D \ln \left[1+e^{\beta\left(\mu-\varepsilon_{m}\right)}\right]$. We have $\left(\partial \Omega_{T}^{m} / \partial B\right)_{\mu}=\Omega_{T}^{m} / B+$ $D\left(\partial \varepsilon_{m} / \partial B\right)\left[1+e^{-\beta\left(\mu-\varepsilon_{m}\right)}\right]^{-1}$, so the conduction magnetization $M_{C, T}=-\mathcal{A}^{-1}\left(\partial \Omega_{C, T} / \partial B\right)_{\mu}$ is given by

$$
M_{C, T}=\frac{K T D}{\mathcal{A} B} \sum_{m=0}^{\infty} \ln \left[1+e^{\beta\left(\mu-\varepsilon_{m}\right)}\right]-\frac{D}{\mathcal{A}} \sum_{m=0}^{\infty} \frac{\partial \varepsilon_{m}}{\partial B} \frac{1}{1+e^{-\beta\left(\mu-\varepsilon_{m}\right)}}
$$




\section{Magnetic oscillations in graphene with spin splitting: a new approach}

We now assume a Fermi energy $\varepsilon_{f}<\mu \leq \varepsilon_{f+1}$, so the conduction magnetization at zero temperature is $M_{C}=-\mathcal{A}^{-1}\left(\partial \Omega_{C} / \partial B\right)_{\mu}=-\mathcal{A}^{-1} \sum_{m=0}^{f}\left[D\left(\varepsilon_{m}-\mu\right) / B+D\left(\partial \varepsilon_{m} / \partial B\right)\right]$. Thus we can rewrite equation $(19)$ as

$$
\begin{aligned}
M_{C, T}=M_{C}+ & \frac{K T D}{\mathcal{A} B} \sum_{m=0}^{f} \ln \left[1+e^{-\beta\left(\mu-\varepsilon_{m}\right)}\right]+\frac{D}{\mathcal{A}} \sum_{m=0}^{f} \frac{\partial \varepsilon_{m}}{\partial B} \frac{1}{1+e^{\beta\left(\mu-\varepsilon_{m}\right)}} \\
& +\frac{K T D}{\mathcal{A} B} \sum_{m=f+1}^{\infty} \ln \left[1+e^{\beta\left(\mu-\varepsilon_{m}\right)}\right]-\frac{D}{\mathcal{A}} \sum_{m=f+1}^{\infty} \frac{\partial \varepsilon_{m}}{\partial B} \frac{1}{1+e^{-\beta\left(\mu-\varepsilon_{m}\right)}} .
\end{aligned}
$$

We can simplify equation 20 by noticing that the term with the logarithm is in general very small. Indeed, we always have $\ln \left[1+e^{-\beta\left(\mu-\varepsilon_{m}\right)}\right] \leq \ln 2<1$ if $m \leq f$ and likewise $\ln \left[1+e^{\beta\left(\mu-\varepsilon_{m}\right)}\right]<\ln 2$ if $m>f$. Furthermore, we have $k T D / \mathcal{A} B=2 k T / \phi=$ $2 k T e / h \sim 10^{-7} T[\mathrm{~K}] \mathrm{meV} / \mathrm{TA}^{2}$, which for low $T$ is much smaller than the magnetization at zero temperature (see figure 1). On the other hand, at low temperatures each exponential term in equation (20) is in general very small unless $\mu$ is close to $\varepsilon_{m}$. Thus we can expand each $\varepsilon_{m}$ around $B_{m}$, where $B_{m}^{-1}=n_{m} / \omega+s_{m} \Delta$ and $\varepsilon_{m}\left(B_{m}\right)=\mu$, so $\left(\mu-\varepsilon_{m}\right) \simeq-\mu\left(B-B_{m}\right) / 2 B_{m}$. Moreover, $\left(\partial \varepsilon_{m} / \partial B\right) \simeq\left[\mu-s_{m} \mu_{\mathrm{B}} B\right] / 2 B$, so from equation 10 we get $\mathcal{A}^{-1}\left(\partial \varepsilon_{m} / \partial B\right) \simeq-A_{s_{m}} / D$. Finally, if we take into account the valence contribution to the magnetization (which we show it is not altered by the temperature), we have the total magnetization $M_{T}=M_{V, T}+M_{C, T}=M_{V}+M_{C, T}$. Thus from equation 20 we get

$$
M_{T} \simeq M-\sum_{m=0}^{f} \frac{A_{s_{m}}}{1+e^{-\beta \mu\left(\vec{B}-B_{m}\right) / 2 B_{m}}}+\sum_{m=f+1}^{\infty} \frac{A_{s_{m}}}{1+e^{\beta \mu\left(B-B_{m}\right) / 2 B_{m}}} .
$$

where $M=M_{V}+M_{C}$ is the net magnetization at zero temperature, given by equation (13). Equation (21) corresponds to the case $\varepsilon_{f}<\mu \leq \varepsilon_{f+1}$, which implies $B_{f+1} \leq B<B_{f}$. Thus the low temperature effect is to introduce a factor proportional to $\left[1+e^{-\beta \mu\left(B-B_{m}\right) / 2 B_{m}}\right]^{-1}$ if $B<B_{m}$, or proportional to $\left[1+e^{\beta \mu\left(B-B_{m}\right) / 2 B_{m}}\right]^{-1}$ if $B>B_{m}$. In this way we can generalize equation (21) to all $B$ and get the result (see the appendix for details)

$$
M_{T}=\sum_{s} \frac{A_{s}}{\pi} \arctan \left\{\cot \left[\pi \omega\left(\frac{1}{B}+s \Delta\right)+\sum_{n} \frac{\pi}{1+e^{-\beta \mu\left(B-B_{n, s}\right) / 2 B_{n, s}}}\right]\right\},
$$

where $B_{n, s}^{-1}=n / \omega-s \Delta$. In the practice it is sufficient to take only the $B_{n}$ between the range of magnetic fields considered.

In figure 4 it is show the magnetization given by equation 22 for $\mu=250 \mathrm{meV}$ and temperatures (a) $T=0.1 \mathrm{~K}$, (b) $T=1 \mathrm{~K}$. We can see that as the temperature increases, the magnetization at $T \neq 0$ broadens, similar to the Fermi-Dirac distribution with the DOS. Of course, this is exactly what is expected from the result given by equation (22). Moreover, in figure 4(a) we see that at very low temperature $T=0.1 \mathrm{~K}$ one can still appreciate the effect of the spin splitting in the magnetization, which results in a small 
1

2

3

4

5

6

7

8

9

Magnetic oscillations in graphene with spin splitting: a new approach
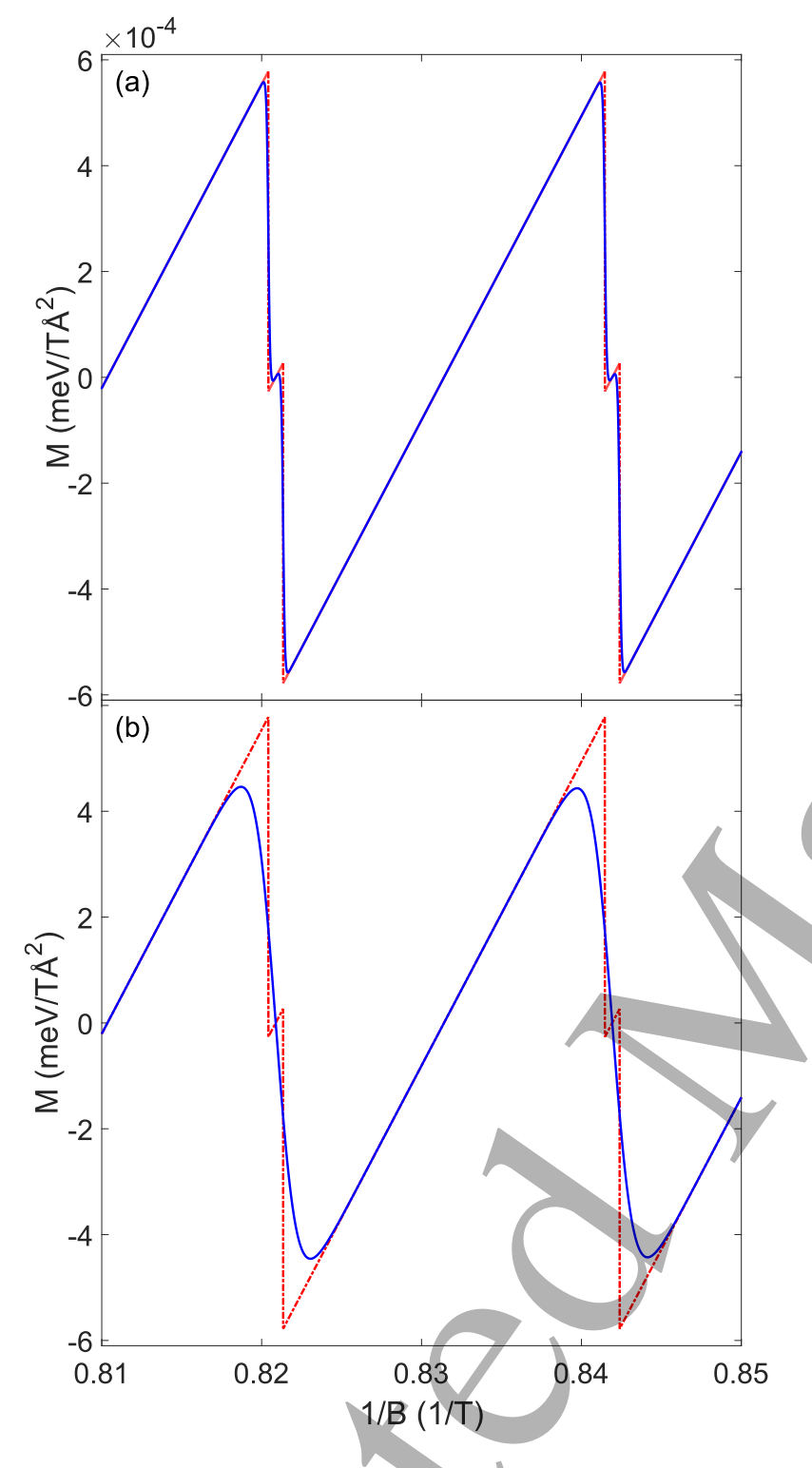

Figure 4. Magnetization given by equation (22), for $\mu=250 \mathrm{meV}$. The red dashed line corresponds to the zero temperature case, while the solid blue line corresponds to (a) $T=0.1 \mathrm{~K}$ and (b) $T=1 \mathrm{~K}$.

bump around the two peaks. Nevertheless, as the temperature is increased, this bump disappears and the MO behave as maxima and minima around the center of the two peaks at zero temperature. Thus in this regime there would be no noticeable effect of the spin splitting in the magnetization. This is strongly dependent not only on the temperature, but also on the magnetic field and Fermi energy. In other words, for high magnetic fields, the temperature at which the spin splitting is negligible increases. It is also worth noting that the introduction of disorder in the system increases the damping of the MO, which would make it even harder to observe the spin splitting. 
Magnetic oscillations in graphene with spin splitting: a new approach

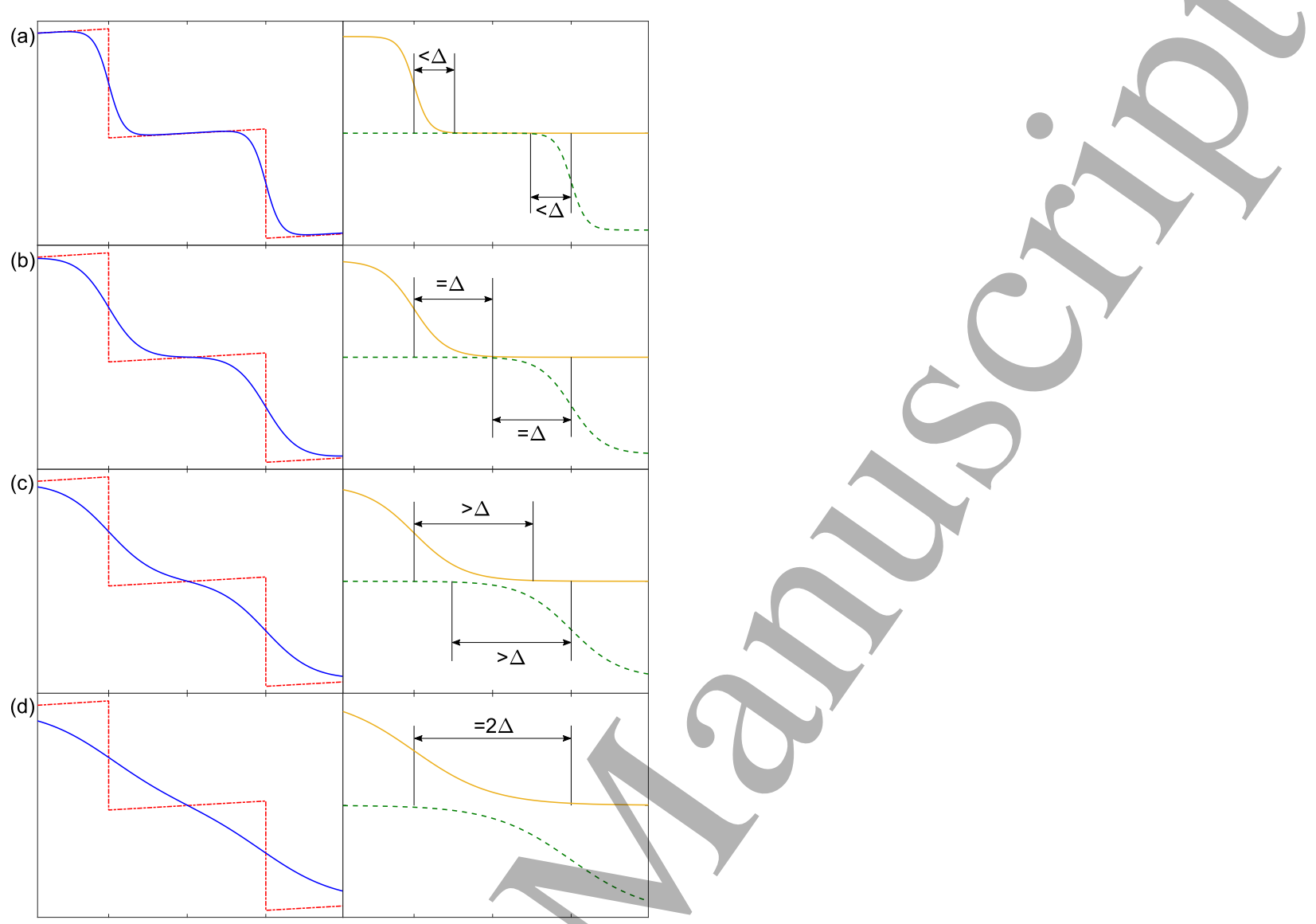

Figure 5. Spin splitting effect in the MO. In the left it is the magnetization at zero temperature (red dashed line) and at different temperatures (blue solid line). In the right it is plotted the first (orange solid line) and second (green dashed line) exponential in equation (23). The four cases correspond to the exponentials width being (a) $<\Delta$, (b) $=\Delta,(\mathrm{c})>\Delta,(\mathrm{d})=2 \Delta$, where $2 \Delta$ is the spin peaks separation.

\subsection{Spin splitting in the MO at nonzero temperature}

An estimation for the temperature at which the spin splitting would not be observable in the MO can be obtained from equation (22). We shall define $x \equiv 1 / B$, and consider two peaks at $x_{1}$ and $x_{2}$, corresponding to the same LL, with different spin, so in general $x_{1}=l / \omega-\Delta$ for the spin up, and $x_{2}=l / \omega+\Delta$ for the spin down (see equation (7)). Then, between the two peaks, only the exponential terms corresponding to $x_{1}$ and $x_{2}$ would be appreciable, so for $x_{1}<x<x_{2}$ equation (22) becomes

$$
M_{T}=M-\frac{A_{1}}{1+e^{\beta \mu\left(x-x_{1}\right) / 2 x}}+\frac{A_{-1}}{1+e^{-\beta \mu\left(x-x_{2}\right) / 2 x}},
$$

where $M$ is given by equation (13), and we have rewrite $\left(B-B_{m}\right) / 2 B_{m}=$ $-\left(x-x_{m}\right) / 2 x$.

From equation (23) we can see that the temperature effect behaves like a FermiDirac distribution. The difference is that in this case our parameter is $x=1 / B$, instead of the energy, and each exponential is center around the position of the peak $x_{m}$. 
Thus we can write $\beta \mu\left(x-x_{m}\right) / 2 x=\tilde{\beta}\left(x-x_{m}\right)$, where $\tilde{\beta}=1 / k \tilde{T} \equiv \beta \mu / 2 x$ can be considered the corresponding temperature parameter, with defines the broadening of each exponential term in equation (23). This shows that the broadening depends not only on the real temperature $T$, but also on the Fermi energy $\mu$ and the magnetic field $x=1 / B$. In this way we can get an estimation to how the temperature affects the observation of the spin splitting in the magnetization, which is dictated by how the width of the exponentials relates to the spin peaks separation $x_{2}-x_{1}=2 \Delta$. This can be seen in figure 5, where for different temperatures we plotted the magnetization (left) and the exponentials that appear in equation (23) (right). Then we can identify four situations. In the first case, figure 5(a), the width is $<\Delta$ and one can clearly appreciate the spin splitting in the magnetization at $T \neq 0$. The second case, figure 5 (b), corresponds to the case when the width is $\Delta$, so the exponentials began to overlap. Then, for a width $>\Delta$ (but $<2 \Delta$ ) in figure $5(\mathrm{c})$, the spin splitting effect starts to disappear, although one could still notice a small change of curvature in $M_{T}$. Finally, when the width is $\geq 2 \Delta$ as in figure 5 (d), the spin splitting becomes unobservable in $M_{T}$, and one is left with what appears as one unique oscillation around the center $\left(x_{1}+x_{2}\right) / 2$.

Now, for a Fermi-Dirac distribution of the form $\left[1+e^{\left(y-y_{0}\right) / a}\right]^{-1}$, the width $w$ around $y_{0}$ is determined by the condition $1 /\left[1+e^{w / a}\right]=\sigma$, where $\sigma \ll 1$ is the appreciation considered. From our experience, it is sufficient to take $\sigma \sim 10^{-2}$, which implies a width $w \sim 5 a$. Therefore, following figure 5(d), the critical temperature for which the spin splitting becomes unobservable satisfies $2 \Delta \simeq 5 k \tilde{T}$. Given that $k \tilde{T}=2 k T x / \mu$, and $2 \Delta=4 \mu_{\mathrm{B}} / \mu$, we get the temperature $T_{s}=2 \mu_{\mathrm{B}} / 5 k x$. This result basically means that, in order of magnitude, the thermal energy $k T$ equals the spin splitting energy $2 \mu_{\mathrm{B}} / x$. One should replace in $x$ the value at which the broadening is computed. If the peak at $x_{1}$ is considered, the corresponding exponential factor is $\tilde{\beta}\left(x-x_{1}\right)$, and the condition $2 \Delta \simeq 5 k \tilde{T}$ implies to take $x=x_{2}$. On the other hand, if we take the peak $x_{2}$, we should take $x=x_{1}$. This gives two different temperatures $T_{s, 1} \simeq 2 \mu_{\mathrm{B}} / 5 K x_{2}$ and $T_{s, 2} \simeq 2 \mu_{\mathrm{B}} / 5 K x_{1}$, but $\left|T_{s, 1}-T_{s, 2}\right|=T_{s, 1}\left(2 \Delta / x_{1}\right)=T_{s, 2}\left(2 \Delta / x_{2}\right)$ with $\Delta / x_{i} \ll 1$ for both cases. Thus in general $T_{s, 1} \simeq T_{s, 2}$, and we can take the average temperature $T_{s}=\left(T_{s, 1}+T_{s, 2}\right) / 2$, so

$$
T_{s} \simeq \frac{2}{5} \frac{\mu_{\mathrm{B}} B}{k},
$$

where $B=\omega / l$ is the center of the spin peaks. Equation 24 gives the maximum temperature to observe the spin splitting in the MO. Given that $B=\omega / l=\mu^{2} / l \alpha^{2}$, then $T_{s}$ is proportional to $\mu^{2}$, so high Fermi energy favors the observation of the spin splitting in the magnetization. It should be noted that the condition given by equation (24) has to be taken into account alongside with the need of high $\mu$ (about $\mu \gtrsim 100 \mathrm{meV}$, see figure 1) to observe the spin splitting at zero temperature. Therefore, for practical terms, equation (24) should only be applied when $\mu \gtrsim 100 \mathrm{meV}$.

In figure 6 we plotted equation (24) as a function of the magnetic field $B$ (solid line) and for $\mu=250 \mathrm{meV}$ (scatter), where $B=\omega / l=\mu^{2} / l \alpha^{2}$. We see that, in general, $T_{s}$ is very low unless $B$ is very high. For instance, at $\mu=250 \mathrm{meV}$, we have that $B \sim 1 \mathrm{~T}$ 
Magnetic oscillations in graphene with spin splitting: a new approach

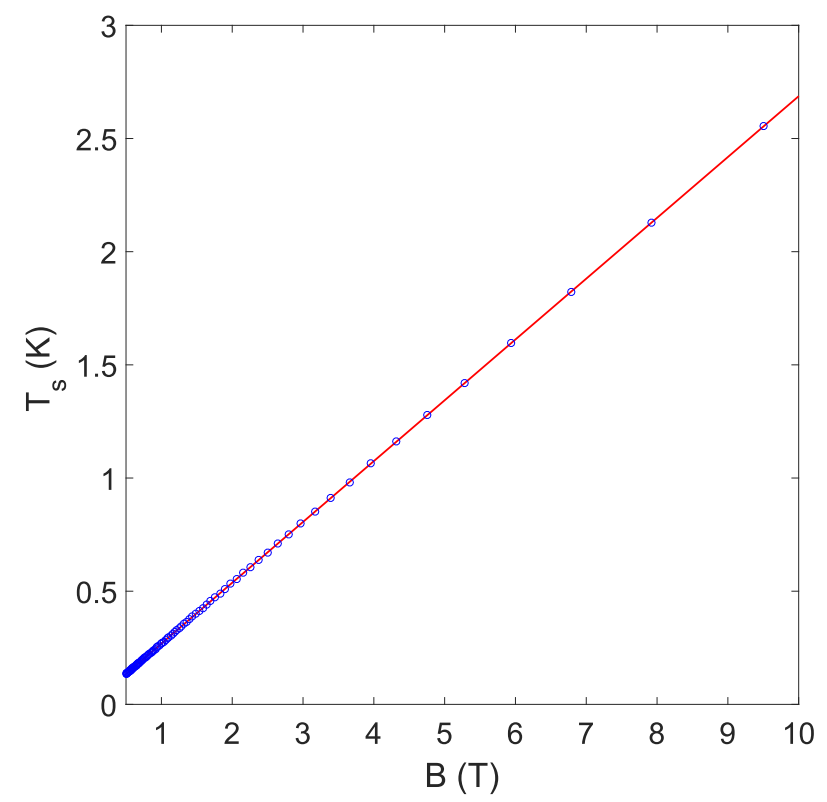

Figure 6. Spin temperature $T_{s}$, given by equation (24), as a function of the magnetic field $B$ (solid line) and for $\mu=250 \mathrm{meV}$ (scatter), where $B=\omega / l=\mu^{2} / l \alpha^{2}$ at the center of the spin peaks corresponding to the LL $l$.

gives $T_{s} \sim 0.3 \mathrm{~K}$. Thus, even for high magnetic field, it requires a very low temperature to observe the spin splitting in the MO. Furthermore, if one also considers disorder in graphene, one should expect that the spin temperature becomes lower with increasing disorder.

\section{2. $M O$ extrema shift}

Once the temperature is such that $T>T_{s}$ in equation (24), the spin splitting becomes unobservable. Then the MO behave as if the spin splitting is neglected, with the oscillation been around $B=\omega / l$. This is just the usual behavior when the MO are studied in graphene without considering the Zeeman effect. Thus, because we shall take $T>T_{s}$ from now on, we will neglect the spin splitting. Then, what one observe is, essentially, that the MO are broadened and reduced as the temperature increases. This reduction depends on the temperature, as well as the magnetic field and Fermi energy. The higher the magnetic field, the more temperature it takes to reduce the MO, and vice versa. From this pattern we can analyze different parameters in the MO, in which case equation (22) will prove useful.

First of all, notice that as the MO are broadened, the maxima and minima of the oscillations shift from $x_{n} \equiv B_{n}^{-1}=n / \omega$ (see equation (7), neglecting the spin splitting) to $x_{n}-p \delta$, where $p=1(-1)$ for the maxima (minima) and $\delta=\delta(T, B, \mu)$ is the shift parameter which in general depends on the three variables $T, B$ and $\mu$. For a fixed $\mu$, we can obtain the function $\delta$ by the extrema condition $\partial M_{T} / \partial x=0$, where $x=1 / B$. 

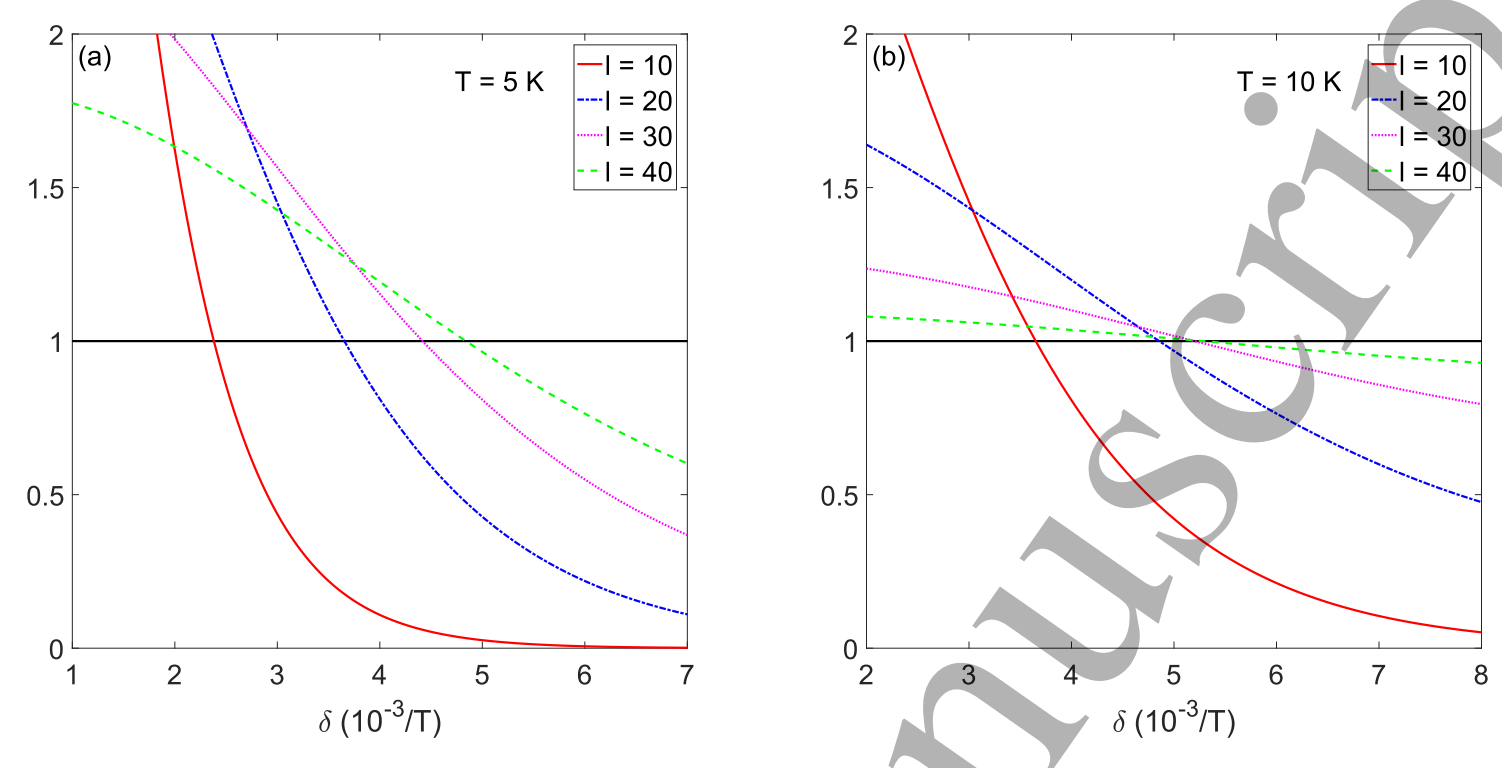

Figure 7. Graphical solution of equation 27) for different LL $l$ and temperatures (a) $T=5 \mathrm{~K}$, (b) $T=10 \mathrm{~K}$. All cases correspond to $\mu=250 \mathrm{meV}$ and maxima shift, so $p=1$ in equation (27).

Now, neglecting the spin splitting, equation (22) becomes

$$
M_{T}=-\frac{2 \mu}{\pi \phi} \arctan \left[\cot \left(\pi \omega x+\sum_{n} \pi f_{n}\right)\right],
$$

where we defined $f_{n} \equiv\left[1+e^{\beta \mu\left(x-x_{n}\right) / 2 x}\right]^{-1}$. Then, the extrema condition $\partial M_{T} / \partial x=0$ can be written as

$$
1=\frac{\beta \mu}{8 \omega x^{2}} \sum_{n} x_{n} \operatorname{sech}^{2}\left[\frac{\beta \mu\left(x-x_{n}\right)}{4 x}\right]
$$

The $x=1 / B$ that satisfy equation $(26)$ give all the new maxima and minima in the MO at $T \neq 0$. Notice that at zero temperature $\beta \rightarrow \infty$ and equation (26) implies $x \rightarrow x_{n}$, as expected. In general, for a peak at $x_{l}=l / \omega$, to obtain its shift it is sufficient to consider only the $n$ close to $l$ (say $l-2<n<l+2$ ) in the summation of equation (26). The new extrema will belocated at $x=x_{l}-p \delta_{l}$, where $\delta_{l}$ is the corresponding shift and $p=1(-1)$ for the maxima (minima). In this way we can rewrite equation 26 as a function of $\delta_{l}$ and the considered peak $x_{l}$ :

$$
1=\frac{\beta \mu}{8 \omega\left(x_{l}-p \delta_{l}\right)^{2}} \sum_{n} x_{n} \operatorname{sech}^{2}\left[\frac{\beta \mu\left(x_{l}-x_{n}-p \delta_{l}\right)}{4\left(x_{l}-p \delta_{l}\right)}\right] .
$$

In figure 7 it is show the graphical solution of equation (27) for different values of temperature and $l$, for $\mu=250 \mathrm{meV}$ and $p=1$ (maxima shift).

As we can see, the shift $\delta$ clearly depends on the LL $l$ and thus on the magnetic field. It increases with the temperature and decreases with the magnetic field. This means that 
Magnetic oscillations in graphene with spin splitting: a new approach

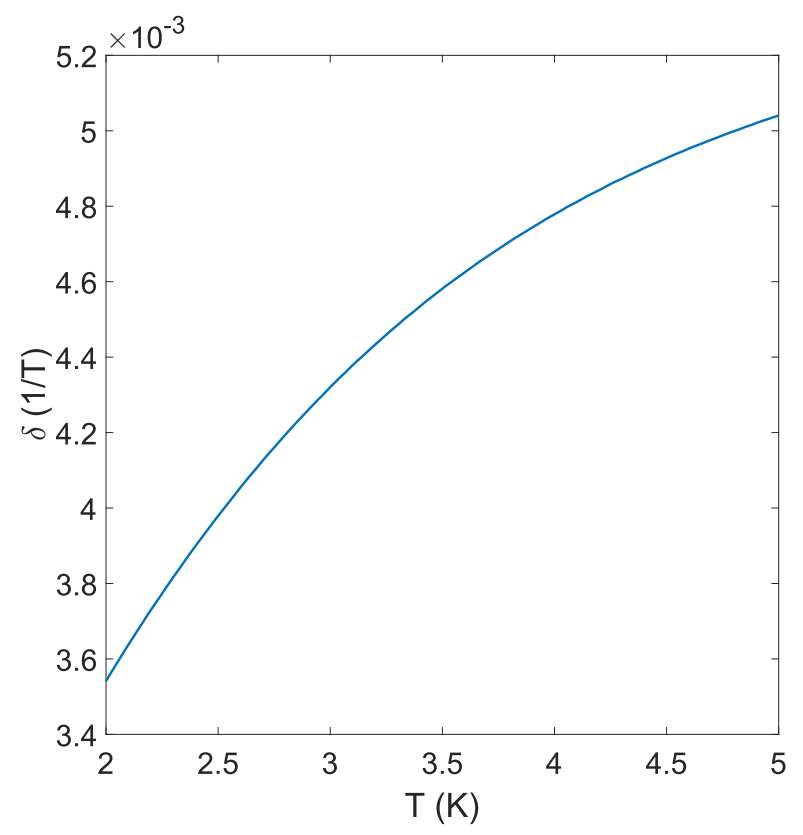

Figure 8. Numerical solution of equation (27) for $\delta_{l}$ as a function of the temperature, where $\mu=250 \mathrm{meV}$ and $B=1 / x_{l}=\omega / l=1.01 \mathrm{~T}$ (corresponding to LL $l=47$ ).

for non zero temperature, the $\mathrm{MO}$ are not anymore periodic in $1 / B$. Indeed, $\delta$ as defined is always measured from the position of the peaks at zero temperature, whose distance between one another is always the same and identical to $1 / \omega$ (the period). Consequently, if at $T \neq 0$ the shift $\delta_{l}$ is not equal for all $l$, then the extrema separation will not be constant and thus not periodic as a function of $1 / B$. Nevertheless, it should be notice that this effect is very small, because for close $l_{1}$ and $l_{2}$ one usually has $\left|\delta_{1}-\delta_{2}\right| \ll 1 / \omega$. On the other hand, in figure 7(b) we see that for the cases $l=30$ and $l=40$, the shift $\delta$ reaches an steady value of about $\delta \sim 5.3 \times 10^{-3} \mathrm{~T}^{-1}$. This means that the extrema in the $\mathrm{MO}$ will tend to be located between the peaks at zero temperature and the zeros in the magnetization. Indeed, the zeros in the MO occur at $B_{0 n}^{-1}=(2 n+1) / 2 \omega$. Thus, the distance between the peaks and the zeros is $\left(B_{n}^{-1}-B_{0 n}^{-1}\right)=1 / 2 \omega$, which is about $\sim 1.05 \times 10^{-2} \mathrm{~T}^{-1}$ for $\mu=250 \mathrm{meV}$ (as considered in figure 7), so the midpoint distance is about $\sim 5.3 \times 10^{-3} \mathrm{~T}^{-1}$.

We can also analyze the shift as a function of the temperature, for a particular LL. This can be seen in figure 8 , which shows the numerical solution of equation (27), for $\mu=250 \mathrm{meV}$ and $B=1 / x_{l}=\omega / l=1.01 \mathrm{~T}$ (corresponding to LL $l=47$ ). As expected, we see that a low temperature $\delta_{l}$ tends to zero, and increases with $T$. This behavior could be useful to measure temperature changes from the MO.

\subsection{MO envelope}

If, at a given temperature, $\delta$ is know as a function of $B$, then the envelope of the MO can be easily obtained. We start considering $x_{1}<x<x_{2}$, where $x_{1}$ and $x_{2}$ are two 
adjacent peaks at zero temperature, so in general $x_{1}=(l-1) / \omega$ and $x_{2}=l / \omega$. Then, from equation 25) we can write

$$
M_{T} \simeq M+\frac{2 \mu}{\phi}\left(\sum_{n=0}^{l-1} \frac{1}{1+e^{\beta \mu(x-n / \omega) / 2 x}}-\sum_{n=l}^{\infty} \frac{1}{1+e^{-\beta \mu(x-n / \omega) / 2 x}}\right)
$$

where $M$ is given by equation (13), which without spin splitting is $M=$ $-2 \mu \arctan [\cot (\pi \omega x)] / \pi \phi$. To obtain the positive envelope corresponding to the maxima in the MO, we just have to eliminate the oscillatory part in (28). For $M$, this can be done by replacing $x=l / \omega-\delta$, the position at which the maximum occurs, where $\delta$ is the shift obtained from equation (27). Thus from equation (13) we get

$$
M_{\delta}=\frac{2 \mu}{\pi \phi} \arctan [\cot (\pi \omega \delta)]
$$

where we defined $M_{\delta} \equiv M(l / \omega-\delta)$. Notice that $M_{\delta}$ is independent of $l$. On the other hand, for the exponentials in equation (28), we need to replace $x=l / \omega-\delta$ only in the numerator. Thus, defining $m=l-n$ we get

$$
\begin{gathered}
{\left[\sum_{m=1}^{l}\left(\frac{1}{1+e^{\beta \mu(m / \omega-\delta) / 2 x}}-\frac{1}{1+e^{\beta \mu(m / \omega+\delta) / 2 x}}\right)-\frac{1}{1+e^{\beta \mu \delta / 2 x}}\right.} \\
\left.-\sum_{m=l+1}^{\infty} \frac{1}{1+e^{\beta \mu(m / \omega+\delta) / 2 x}}\right]
\end{gathered}
$$

The last term in equation (30) can be neglected because it is very small if $x_{1}<x<x_{2}$. In this way, regrouping the exponentials we get the following expression for the positive envelope

$$
\begin{aligned}
& E_{+}=\frac{2 \mu}{\pi \phi}\left\{\arctan [\cot (\pi \omega \delta)]-\frac{\pi}{1+e^{\beta \mu \delta / 2 x}}\right. \\
& \left.+\sum_{m \geq 1} \frac{\pi \sinh [\beta \mu \delta / 2 x]}{\cosh [\beta \mu \delta / 2 x]+\cosh [\beta \mu m / 2 \omega x]}\right\} \text {. }
\end{aligned}
$$

It should be notice that although this envelope was originally obtained for $x_{1}<x<x_{2}$, the expression given by equation (31) is directly generalized and valid for all magnetic fields. The negative envelope is just $E_{-}=-E_{+}$. Although there is a summation involved in $E_{+}$, in the practice it is sufficient to consider only the first few terms. In fact, the terms $m>1$ give corrections only if we are at high temperature and/or low magnetic field, at which the $\mathrm{MO}$ are small.

The envelope given by equation (31) depends on the shift $\delta$, which in general depends on the magnetic field, as it was shown in the last section. A numerical solution for $\delta$ can be obtained from equation (27). This gives $\delta_{l}$ as a function of $l$, from which the function $\delta(x)$ can be obtained by replacing $x=l / \omega$ and fitting the points. This procedure is done in figure 9 , for $T=5 \mathrm{~K}$ and $\mu=250 \mathrm{meV}$, where $\delta$ was fitted 
Magnetic oscillations in graphene with spin splitting: a new approach

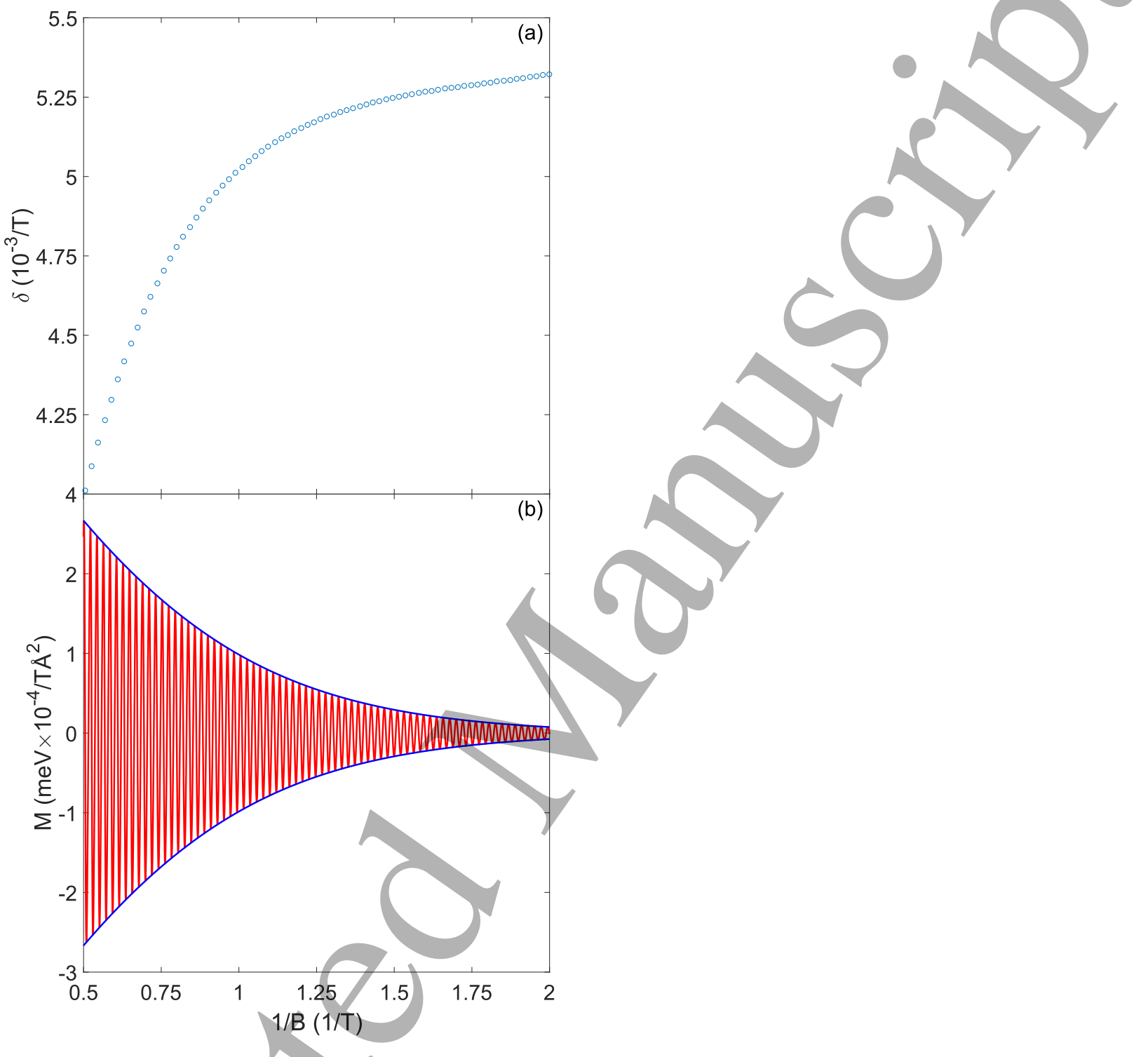

Figure 9. For $T=5 \mathrm{~K}$ and $\mu=250 \mathrm{meV}$ : (a) Numerical solution of equation 27), where $\delta(x)$ is obtained by replacing $x=l / \omega$. The points were fitted with a Boltzmann function. (b) $\mathrm{MO}$ with the positive $E_{+}$and negative $E_{-}=-E_{+}$envelope, where $E_{+}$ is given by equation (31). The shift function $\delta(B)$ used was obtained from the fitting of $(\mathrm{a})$.

with a Boltzmann function. Notice that the figure $9(\mathrm{a})$ is in agreement with the figure 7. where $\delta$ increases with $l$ and thus with $1 / B=x=l / \omega$, and it tends to the limit $\delta \sim 5.3 \times 10^{-3} \mathrm{~T}^{-1}$. Once obtained $\delta(x)$, the positive envelope as a function of $x$ is given by equation (31), while the negative envelope is $E_{-}=-E_{+}$. This can be seen in figure 9 (b), where the MO and the envelopes are shown. We consider only the terms $m=1,2$ in the sum of equation (31), which shows that only the few first terms are needed. 


\section{Conclusions}

We analyzed the magnetic oscillations (MO) in pristine graphene, under a perpendicular magnetic field and taking into account the Zeeman effect. For a constant Fermi energy $\mu>0$ and zero temperature we showed that, due to the Zeeman effect, the MO consist of two sawtooth peaks, each corresponding to one spin. Both peaks have the same frequency, but different amplitude and phase. We obtaining that it requires high Fermi energy, about $\mu \gtrsim 0.1 \mathrm{eV}$.

At $T \neq 0 \mathrm{~K}$, the temperature effect is usually considered by introducing a reduction factor. Nevertheless, the resulting infinite series cannot be evaluated, and can only be approximated for special cases like low $B$, which in turn difficult the observation of the MO. Hence, we took another route by going back to the grand potential. From this we obtained that the MO at low $T$ can be expressed as the MO at zero temperature, plus small correction functions. These functions are Fermi-Dirac like, each centered around the MO peaks at zero temperature. Moreover, they are very small unless the magnetic field is close to the corresponding peaks, which can be useful from a practical sense. Using this expression we then analyzed how the temperature affects the observation of the spin splitting in the MO. We show that, in order of magnitude, the observation is possible when the thermal energy $k T$ is less than the spin splitting energy $2 \mu_{\mathrm{B}} B$. One would also expect that any kind of disorder would hide even further the spin splitting observation in the MO.

We also analyzed the shift of the MO extrema as the temperature increases. We obtained an expression which was solved numerically, showing that the shift increases with the temperature and decreases with the magnetic field, implying that the MO are not anymore periodic at $T \neq 0$. This behavior could be useful to measure temperature changes from the MO. For instance, one possibility would be a graphene device which measures the MO around a particular magnetic field. Then, by analyzing the extrema shift from its known value at zero temperature, one can infer the temperature. Finally, we obtained an analytical expression for the MO envelope, which in turn depends on the shift of the extrema.

\section{Acknowledgments}

This paper was partially supported by grants of CONICET (Argentina National Research Council) and Universidad Nacional del Sur (UNS) and by ANPCyT through PICT 2014-1351. Res. N 270/15. N: 2014-1351, and PIP 2014-2016. Res. N 5013/14. Código: 11220130100436CO research grant, as well as by SGCyT-UNS., J. S. A. and P. J. are members of CONICET., F. E. acknowledge research fellowship from this institution. 
Magnetic oscillations in graphene with spin splitting: a new approach

\section{Appendix}

We shall prove that equation $(22)$ is in agreement with equation (21). First of all, we recall the following properties for $x \in \mathbb{R}$ :

$$
\begin{aligned}
& \cot x=\tan (\pi / 2-x) \\
& \arctan [\tan (x)]=x-\pi \text { floor }\left\{\frac{x+\pi / 2}{\pi}\right\},
\end{aligned}
$$

where floor \{\} is the floor function. Thus, taking only one spin, we can write equation 22) as

$$
\frac{M_{T, s}}{A_{s}}=\frac{1}{2}-\omega\left(\frac{1}{B}+s \Delta\right)-\sum_{m} g_{m}-\text { floor }\left\{1-\omega\left(\frac{1}{B}+s \Delta\right)-\sum_{m} g_{m}\right\},
$$

where we have defined $g_{m}=\left[1+e^{-\beta \mu\left(B-B_{m}\right) / 2 B_{m}}\right]^{-1}$, with $B_{m}^{-1}=n_{m} / \omega-s_{m} \Delta$. Considering $\varepsilon_{f}<\mu \leq \varepsilon_{f+1}$, which implies $B_{f+1} \leq B<B_{f}$, we have

$$
g_{m}= \begin{cases}<1 / 2 & \text { for } m \leq f \\ \geq 1 / 2 & \text { for } m \geq f+1\end{cases}
$$

Furthermore, at low temperatures $g_{m} \ll 1$ if $m<f$, while $g_{m} \rightarrow 1$ if $m>f+1$. These considerations, along with the properties of the floor function, implies that for $B_{f+1} \leq B<B_{f}$, equation A.2 can be written as

$$
\begin{aligned}
\frac{M_{T, s}}{A_{s}}=\frac{1}{2}-\omega & \left(\frac{1}{B}+s \Delta\right)-\text { floor }\left\{1-\omega\left(\frac{1}{B}+s \Delta\right)\right\} \\
& -\sum_{m \leq f} \frac{1}{1+e^{-\beta \mu\left(B-B_{m}\right) / 2 B_{m}}}+\sum_{m \geq f+1} \frac{1}{1+e^{\beta \mu\left(B-B_{m}\right) / 2 B_{m}}} .
\end{aligned}
$$

Consequently, from equation (13) and the properties given by equation (A.1), equation A.4 becomes

$$
M_{T, s}=M_{s}-\sum_{m \leq f} \frac{A_{s}}{1+e^{-\beta \mu\left(B-B_{m}\right) / 2 B_{m}}}+\sum_{m \geq f+1} \frac{A_{s}}{1+e^{\beta \mu\left(B-B_{m}\right) / 2 B_{m}}},
$$

so equation 22 effectively reduces to equation 21) if $\varepsilon_{f}<\mu \leq \varepsilon_{f+1}$.

\section{References}

[1] K. S. Novoselov, A. K. Geim, S. V. Morozov, D. Jiang, M. I. Katsnelson, I. V. Grigorieva, S. V. Dubonos, and A. A. Firsov. Two-dimensional gas of massless dirac fermions in graphene. Nature, 438(7065):197-200, nov 2005.

[2] A. K. Geim and K. S. Novoselov. The rise of graphene. Nature Materials, 6(3):183-191, mar 2007.

[3] Yuanbo Zhang, Yan-Wen Tan, Horst L. Stormer, and Philip Kim. Experimental observation of the quantum hall effect and berrys phase in graphene. Nature, 438(7065):201-204, nov 2005. 
Magnetic oscillations in graphene with spin splitting: a new approach

[4] Phaedon Avouris and Christos Dimitrakopoulos. Graphene: synthesis and applications. Materials Today, 15(3):86-97, mar 2012.

[5] K. S. Novoselov, V. I. Fal/ko, L. Colombo, P. R. Gellert, M. G. Schwab, and K. Kim. A roadmap for graphene. Nature, 490(7419):192-200, oct 2012.

[6] P. R. Wallace. The band theory of graphite. Physical Review, 71(9):622-634, may 1947.

[7] A. H. Castro Neto, F. Guinea, N. M. R. Peres, K. S. Novoselov, and A. K. Geim. The electronic properties of graphene. Reviews of Modern Physics, 81(1):109-162, jan 2009.

[8] Ganhua Lu, Kehan Yu, Zhenhai Wen, and Junhong Chen. Semiconducting graphene: converting graphene from semimetal to semiconductor. Nanoscale, 5(4):1353, 2013.

[9] Choongyu Hwang, David A. Siegel, Sung-Kwan Mo, William Regan, Ariel Ismach, Yuegang Zhang, Alex Zettl, and Alessandra Lanzara. Fermi velocity engineering in graphene by substrate modification. Scientific Reports, 2(1), aug 2012.

[10] M. O. Goerbig. Electronic properties of graphene in a strong magnetic field. Reviews of Modern Physics, 83(4):1193-1243, nov 2011.

[11] Juan Sebastian Ardenghi, Pablo Bechthold, Estela Gonzalez, Paula Jasen, and Alfredo Juan. Valley properties of doped graphene in a magnetic field. The European Physical Journal B, 88(2), feb 2015.

[12] J.S. Ardenghi, P. Bechthold, E. Gonzalez, P. Jasen, and A. Juan. Statistical repulsion/attraction of electrons in graphene in a magnetic field. Physica B: Condensed Matter, 433:28-36, jan 2014.

[13] J.S. Ardenghi, P. Bechthold, P. Jasen, E. Gônzalez, and O. Nagel. Landau level transitions in doped graphene in a time dependent magnetic field. Physica B: Condensed Matter, 427:97-105, oct 2013.

[14] F. Escudero, J.S. Ardenghi, and P. Jasen. Magnetic oscillations in silicene. Journal of Magnetism and Magnetic Materials, 454:131-138, may 2018.

[15] §̧ Kuru, J Negro, and L M Nieto. Exact analytic solutions for a dirac electron moving in graphene under magnetic fields. Journal of Physics: Condensed Matter, 21(45):455305, oct 2009.

[16] W. de Haas and P. van Alphen. The dependence of the susceptibility of diamagnetic metals upon the field. Proc.Acad.Sci.Amst, 33(1106-1118), 1930.

[17] S. G. Sharapov, V. P. Gusynin, and H. Beck. Magnetic oscillations in planar systems with the dirac-like spectrum of quasiparticle excitations. Physical Review B, 69(7), feb 2004.

[18] Zhen-Guo Fu, Zhi-Gang Wang, Shu-Shen Li, and Ping Zhang. Magnetic quantum oscillations in a monolayer graphene under a perpendicular magnetic field. Chinese Physics B, 20(5):058103, may 2011.

[19] F. Escudero, J.S. Ardenghi, L. Sourrouille, and P. Jasen. Ground state magnetization of conduction electrons in graphene with zeeman effect. Journal of Magnetism and Magnetic Materials, 429:294-298, may 2017.

[20] D. Shoenberg. Magnetic oscillations in metals. Cambridge University Press, 1984.

[21] R. Hanson, L. M. K. Vandersypen, L. H. Willems van Beveren, J. M. Elzerman, I. T. Vink, and L. P. Kouwenhoven. Semiconductor few-electron quantum dot operated as a bipolar spin filter. Physical Review B, 70(24), dec 2004.

[22] R. M. Potok, J. A. Folk, C. M. Marcus, V. Umansky, M. Hanson, and A. C. Gossard. Spin and polarized current from coulomb blockaded quantum dots. Physical Review Letters, 91(1), jul 2003.

[23] N. Sinitsyn, E. Hankiewicz, Winfried Teizer, and Jairo Sinova. Spin hall and spin-diagonal conductivity in the presence of rashba and dresselhaus spin-orbit coupling. Physical Review $B, 70(8)$, aug 2004.

[24] F. Escudero, L. Sourrouille, J.S. Ardenghi, and P. Jasen. Magnetization in pristine graphene with zeeman splitting and variable spin-orbit coupling. Superlattices and Microstructures, 101:537546, jan 2017.

[25] F. Escudero, J.S. Ardenghi, Paula Jasen, and A. Juan. Impurity effects in the magnetic oscillations on doped graphene with zeeman splitting. Physica B: Condensed Matter, 518:39-46, aug 2017. 
Magnetic oscillations in graphene with spin splitting: a new approach

[26] P. Zeeman. VII.doublets and triplets in the spectrum produced by external magnetic forces. Philosophical Magazine Series 5, 44(266):55-60, jul 1897.

[27] Ming-Che Chang, Min-Fong Yang, and Tzay-Ming Hong. Effect of landau-level mixing for electrons in a random magnetic field. Physical Review B, 56(7):3602-3605, aug 1997.

[28] C J Tabert and J P Carbotte. Magnetization of the metallic surface states in topological insulators. Journal of Physics: Condensed Matter, 27(1):015008, nov 2014.

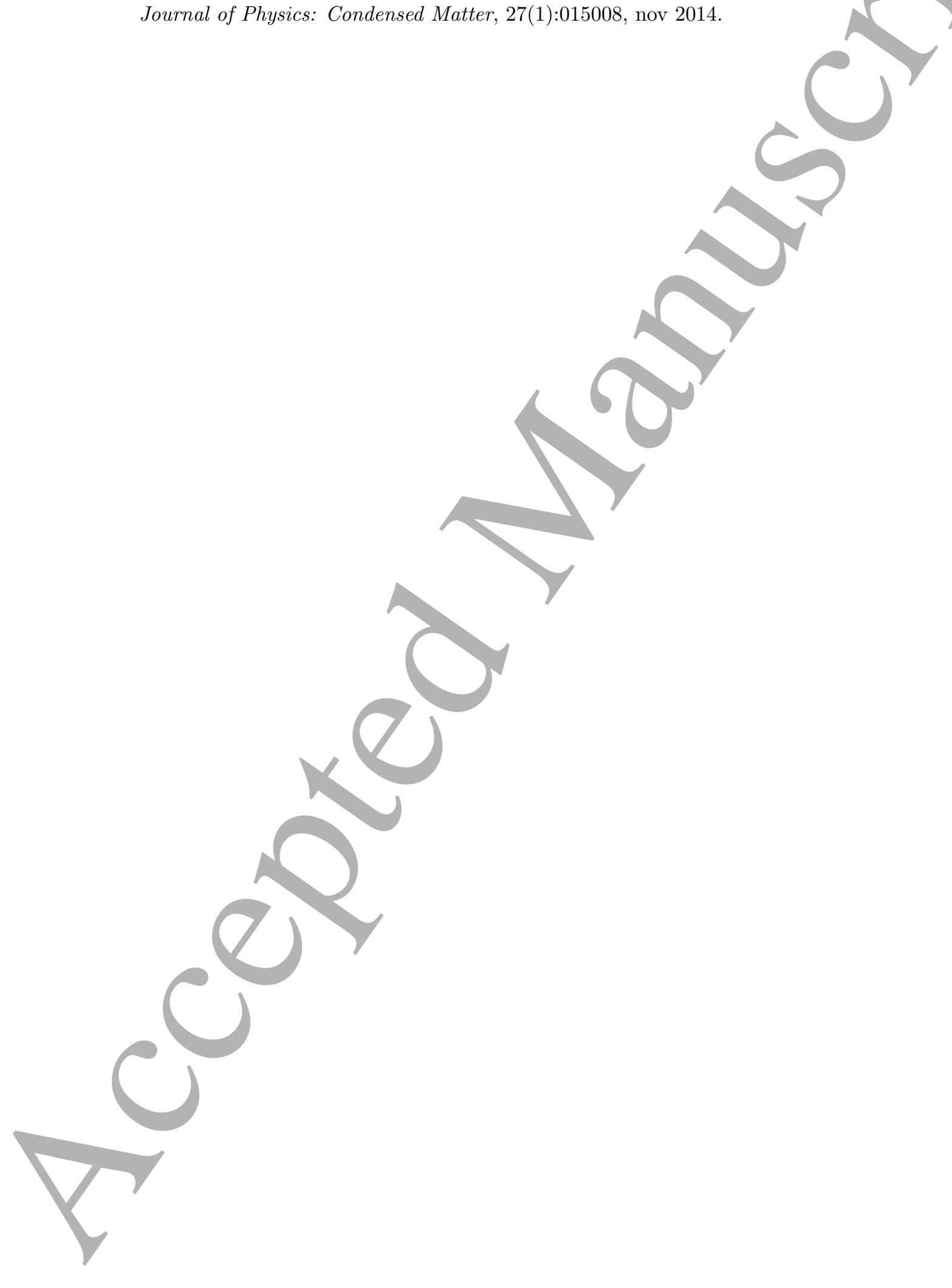

